\title{
Configuração da cobertura de saúde bucal brasileira e o acesso da população ao serviço público odontológico
}

\section{Brazilian oral health coverage characteristics and the populational access to public dental services}

\section{Configuración de la cobertura de salud bucal brasilera y el acceso de la población al servicio público odontológico}

\section{Recebido: $19 / 03 / 2017$ \\ Aprovado: 06/12/2017 \\ Publicado: 05/04/2018}

\section{Raphael Cavalcante Costa ${ }^{1}$ Isabella Lima Arrais Ribeiro ${ }^{2}$ Larycia Vicente Rodrigues ${ }^{3}$ Ana Maria Gondim Valença ${ }^{4}$}

O objetivo deste estudo foi analisar a cobertura de saúde bucal brasileira e o acesso da população ao serviço público odontológico entre os anos de 2008 e 2012. Trata-se de um estudo ecológico, comparativo-descritivo, por documentação indireta. As médias de Equipes de Saúde Bucal (ESB) do serviço público brasileiro variam de acordo com as regiões. No período avaliado, o Nordeste apresentou maior média de ESBs $(64,0)$; seguido das regiões Sul $(56,0)$; Centro-Oeste $(53,0)$; Norte $(44,0)$ e Sudeste $(43,0)$. A região Nordeste concentra as maiores médias populacionais que nunca realizaram consulta odontológica, mas essa é a região que apresenta a maior oferta de serviço odontológico. As menores médias concentram-se no Sul, que após o Nordeste é a região com maior oferta de serviço público odontológico. Verificou-se diferença entre a proporção da população que nunca realizou consulta odontológica e a proporção da população com cobertura de plano de saúde $(p=0,009)$. 0 acesso e a utilização dos serviços públicos de saúde bucal pela população brasileira, nas diferentes regiões geoeconômicas, bem como a quantidade de equipes de saúde bucal é desigual, havendo maior procura pela atenção em saúde bucal entre os usuários que possuem plano de saúde privado, que pertencem ao sexo feminino, e residem na área urbana. Descritores: Estratégia de Saúde da Família; Saúde bucal; Acesso aos serviços de saúde.

The aim of this study was analyzing the Brazilian oral health coverage and the population access to the odontological public service between 2008 and 2012. It is an ecological and comparative-descriptive study, made from indirect records. The means of the Oral Health Teams (OHTs) in the Brazilian public services varied according to the regions. In the evaluated period, the Northeast presented higher OHT means (64.0); followed by the South (56.0); Midwest (53.0); North (44.0) and Southeast (43.0). The Northeast region has the highest number of population means who never underwent odontological consultations, even though this is the region with the highest offer of odontological services. The lowest means are concentrated in the South, which, after the Northeast, has the highest offer of public odontological services. There was a difference between the proportion of people who never underwent odontological consultations and those who have health insurances $(p=0.009)$. The access and use of public oral health services by the Brazilian population, in the different geo-economic regions, as well as the amount of oral health teams is unequal. Those who have private health insurances, are female and live in urban areas are the ones who seek oral health care the most.

Descriptors: Family Health Strategy; Oral health; Health services accessibility.

El objetivo de este estudio fue analizar la cobertura de salud bucal brasilera y el acceso de la población al servicio público odontológico entre los años de 2008 y 2012. Se trata de un estudio ecológico, comparativo-descriptivo, por documentación indirecta. En el periodo evaluado, el Noreste presentó mayor promedio de ESBs $(64,0)$; seguido de las regiones Sur (56,0); Centro-Oeste $(53,0)$; Norte $(44,0)$ y Sureste $(43,0)$. La región Noreste concentra los mayores promedios poblacionales que nunca realizaron consulta odontológica, pero esta es la región que presenta la mayor oferta de servicio público odontológico. Se verificó la diferencia entre la proporción de la población que nunca realizó consulta odontológica y la proporción de la población con cobertura de plan de salud $(\mathrm{p}=0,009)$. El acceso y la utilización de los servicios públicos de salud bucal por la población brasilera, en las diferentes regiones geoeconómicas, así como la cantidad de equipos de salud bucal es desigual, habiendo mayor búsqueda por la atención en salud bucal entre los usuarios que poseen plan de salud privado, que pertenecen al sexo femenino y residen en el área urbana.

Descriptores: Estrategia de Salud Familiar; Salud bucal; Accesibilidad a los servicios de salud.

1 Graduando em Odontologia pela Universidade Federal da Paraíba (UFPB), João Pessoa, PB, Brasil. ORCID: 0000-0002-1333-5227 E-mail: raphaelcavalcante_@hotmail.com

2 Cirurgiã Dentista. Doutora em Modelos de Decisão e Saúde. João Pessoa, PB, Brasil. ORCID: 0000-001-6538-6811 E-mail: isabella_arrais@yahoo.com

${ }^{3}$ Enfermeira. Especialista em Hematologia e Hemoterapia. Especialista em Vigilância Sanitária. Mestre em Modelos de Decisão e Saúde. Doutoranda Modelos de Decisão e Saúde pela UFPB. Enfermeira do Hospital Universitário Dr. Washington Antonio de Barros, João Pessoa, PB, Brasil. ORCID: 0000-0002-4905-8161 E-mail: larycia_rodrigues@hotmail.com

${ }^{4}$ Cirurgião Dentista. Mestre e Doutora em Odontologia. Professora Titular dos Programas de Pós Graduação em Modelos de Decisão e Saúde e, Programa de Pós Graduação em Odontologia e do Departamento de Clínica e Odontologia Social da UFPB, João Pessoa, PB, Brasil. ORCID: 0000-0001-8460-3981 E-mail: anaval@terra.com.br 


\section{INTRODUÇÃO}

A implantação da Estratégia de Saúde da Família (ESF), em 1994, foi uma tentativa de tornar o modelo de saúde mais universal, integral, participativo e descentralizado. Desde sua criação, vem impactando em melhorias na saúde pública do Brasil. Inicialmente, o programa era composto por uma equipe integrada de médicos, enfermeiros, técnicos e agentes comunitários de saúde ${ }^{1}$.

Seguindo o eixo da Integralidade do Sistema Único de Saúde (SUS), bem como a demanda, o aumento do número de profissionais e a sua atuação prévia em alguns programas, o cirurgião-dentista foi admitido a partir de 2000, por meio da portarias no 1.444 e n²67/2001 do Ministério da Saúde, como integrante da equipe multiprofissional do PSF, a qual teve sua prática auxiliada por incentivos financeiros e estruturais para expansão e melhoria da saúde bucal no país ${ }^{2-}$ 4.

Posteriormente, pautado nas diretrizes da Política Nacional de Saúde Bucal (PNSB), e como forma de consolidação da atenção secundária em odontologia, surge em 2004 o Programa Brasil Sorridente, mediante implantação dos Centros de Especialidades Odontológicas (CEOs) ${ }^{5,6}$.

Desde então, a saúde bucal vem atuando na atenção básica e especializada, aproximando a prática odontológica da população mais carente e necessitada. Concomitantemente, vêm melhorando aspectos da prática odontológica, como a diminuição da prevalência de cáries nas dentições decídua e permanente e a consequente redução da perda precoce de dentes, bem como tem sido ampliado o acesso ao diagnóstico precoce de enfermidades bucais ${ }^{7,8}$.

No entanto, ainda se verifica que há fragilidades no acesso aos serviços públicos de saúde bucal, além de dificuldades inerentes ao sistema, como a falta de insumos, ou de serviços ainda não ofertados no âmbito do SUS. Por outro lado, têm ocorrido o aumento no número de usuários cobertos por planos de saúde privados, que, indiretamente, buscam o serviço odontológico por essa via $7,9,10$.
Segundo a Pesquisa Nacional de Amostra por Domicílio (PNAD), esses usuários são desigualmente distribuídos entre as regiões brasileiras, apresentando uma maior concentração na região Sudeste e vivendo predominantemente em domicílios urbanos $^{11}$. Contudo, nem toda a população tem acesso a este tipo de serviço e depende das ações vinculadas ao SUS ${ }^{10}$.

Assim, o objetivo deste estudo foi analisar a cobertura de saúde bucal brasileira e sua relação com o acesso da população ao serviço público odontológico, entre os anos de 2008 a 2012.

\section{MÉTODO}

Realizou-se um estudo ecológico, com abordagem indutiva, procedimento comparativo-descritivo e técnica de documentação indireta ${ }^{12}$. Foram considerados os dados referentes à cobertura de saúde bucal e sua relação com o acesso da população brasileira ao serviço público odontológico.

Seguiram-se as faixas etárias previamente preconizadas pelo sistema de informação, de 0 a 9, 10 a 19, 20 a 39, 40 a 59, 60 anos ou mais, considerando as cinco regiões brasileiras, o sexo, a renda e a localização.

Os dados foram obtidos a partir dos registros contidos no Departamento de Informática do Sistema Único de Saúde (DATASUS) no período de 2008 a 201213. Foram filtrados os dados referentes ao número de Equipes de Saúde Bucal (ESB), à população que nunca realizou consulta odontológica, aos usuários que realizaram a última consulta a menos de um ano, e à taxa populacional coberta por planos privados de saúde.

Após a coleta, o programa Microsoft Office Excel ${ }^{\circledR}$ foi utilizado para tabulação dos dados, que foram posteriormente exportados para o programa IBM SPSS (Statistical Package for Social Sciences), versão 20.0.

Os dados foram tratados e analisados de maneira descritiva - dispondo os resultados obtidos na forma de gráficos e tabelas com frequências absolutas e percentuais - e inferencial, a partir do teste Qui-Quadrado, 
para avaliar a associação entre as variáveis, adotando-se um nível de significância de $\alpha=5 \%$.

Todos os valores encontrados nos bancos de dados são apresentados em médias aritméticas. Como esses são dados secundários, optou-se por manter a medida de dispersão adotada inicialmente, para evitar alterações e distorção dos resultados. A ausência destas informações em outros sistemas de informação também foi um dos fatores de escolha destes dados nessa plataforma.

\section{RESULTADOS}

As médias de ESB do serviço público brasileiro variam de acordo com as regiões. No período de 2000-2012, o Nordeste apresentou uma maior média de $\operatorname{ESBs}(64,0)$; seguido das regiões Sul $(56,0)$; Centro-Oeste $(53,0)$; Norte $(44,0)$; e Sudeste $(43,0)$. Com essa distribuição desigual do serviço odontológico ofertado à população, deve-se levar em consideração a organização e oferta de profissionais, disponibilidade de serviço e quantidade de usuários por região.

Com base nas informações contidas na Tabela 1, pode-se observar que as maiores médias populacionais que referem nunca ter realizado consulta odontológica são, em todas as variáveis, da região Nordeste, que antagonicamente é a região que apresenta uma maior oferta de serviço odontológico.

Essa população analisada é predominantemente do sexo feminino $(19,6)$; de moradia urbana $(26,5)$; com faixa de até 0,74 salário mínimo e frequentemente em qualquer grupo etário. As menores médias concentram-se no Sul, que após o Nordeste é a região que apresenta uma relevante quantidade de ESB.

Tabela 1. Médias populacionais das regiões brasileiras, no período de 2008 a 2012, que nunca realizaram consulta odontológica. De acordo com sexo, faixa etária, localização e renda.

\begin{tabular}{|c|c|c|c|c|c|}
\hline Variáveis & Norte & Nordeste & Sudeste & Sul & Centro Oeste \\
\hline \multicolumn{6}{|l|}{ Sexo } \\
\hline Masculino & 16,0 & 15,5 & 7,8 & 7,0 & 9,1 \\
\hline Feminino & 18,3 & 19,6 & 9,2 & 8,2 & 10,4 \\
\hline \multicolumn{6}{|l|}{ Localização } \\
\hline Urbano & 24,0 & 26,5 & 12,1 & 9,6 & 13,1 \\
\hline Rural & 15,2 & 14,1 & 8,1 & 7,1 & 9,2 \\
\hline \multicolumn{6}{|c|}{ Faixa Etária (anos) } \\
\hline 0 a 9 & 59,3 & 62,2 & 46,7 & 41,0 & 44,4 \\
\hline 10 a 19 & 13,6 & 17,5 & 5,5 & 4,2 & 6,1 \\
\hline 20 а 39 & 3,8 & 5,0 & 1,6 & 1,4 & 2,2 \\
\hline 40 a 59 & 3,3 & 4,2 & 1,3 & 1,5 & 2,0 \\
\hline 60 anos ou mais & 5,7 & 6,5 & 2,6 & 2,6 & 3,6 \\
\hline \multicolumn{6}{|c|}{ Renda Familiar (SM)* } \\
\hline 0 a 0,74 & 23,4 & 22,5 & 15,5 & 15,0 & 15,9 \\
\hline 0,75 a 1,99 & 9,0 & 7,5 & 6,3 & 5,3 & 6,9 \\
\hline 2 ou mais & 4,2 & 3,9 & 2,8 & 2,5 & 3,1 \\
\hline Sem declaração & 15,2 & 15,4 & 5,1 & 6,4 & 8,3 \\
\hline
\end{tabular}

*Salário Mínimo (SM): análise com base no salário mínimo de R \$622,00 correspondente ao período do estudo.

Na Tabela 2, observam-se as médias populacionais dos usuários que realizaram consulta odontológica no intervalo de menos de 1 ano. Em relação à periodicidade do serviço, a busca maior é por parte da população feminina da região Sul $(50,5)$; de moradia urbana $(49,5)$; e em todas as faixas etárias. A população masculina da região Norte é a que apresenta maior ausência do serviço, caracterizando-se como de moradia urbana (34,9); e faixa etária de 60 anos ou mais. 
Tabela 2. Médias populacionais das regiões brasileiras, nos anos de 2008 a 2012, cuja última consulta odontológica foi há menos de um ano, de acordo com sexo, faixa etária e locação.

\begin{tabular}{lccccc}
\multicolumn{1}{c}{ Variáveis } & Norte & Nordeste & Sudeste & Sul & Centro-Oeste \\
\hline Sexo & & & & & \\
\hline Masculino & 30,2 & 31,5 & 39,4 & 45,4 & 39,6 \\
\hline Feminino & 35,6 & 38,0 & 44,7 & 50,5 & 44,9 \\
\hline Locação & & & & & \\
\hline Urbano & 34,9 & 38,4 & 42,6 & 49,5 & 43,5 \\
\hline Rural & 25,8 & 25,4 & 36,6 & 41,1 & 34,5 \\
\hline Faixa Etária (anos) & & & & & 40,8 \\
\hline 0 a 9 & 27,5 & 26,9 & 39,3 & 45,5 & 51,8 \\
\hline 10 a 19 & 43,7 & 44,5 & 53,2 & 62,4 & 47,0 \\
\hline 20 a 39 & 37,8 & 43,5 & 48,2 & 55,1 & 37,2 \\
\hline 60 a 59 & 26,0 & 29,0 & 38,4 & 42,3 & 20,8 \\
\hline 60 anos ou mais & 10,2 & 13,1 & 22,7 & 24,9 & \\
\hline
\end{tabular}

*Salário Mínimo (SM): análise com base no salário mínimo de R\$ 622,00 correspondente ao período do estudo.

Ao se considerar a taxa populacional idade e residentes urbanos, para todas as coberta por planos de saúde (Tabela 3), regiões do país.

verifica-se que há uma ligeira diferença Observou-se que há uma diferença quanto ao sexo, sendo maior o número de significante entre a proporção da população usuários do sexo feminino, comparado ao que nunca realizou consulta odontológica e a masculino; além de uma maior concentração proporção da população com cobertura de de usuários na faixa etária de 40-60 anos de plano de saúde $(\mathrm{p}=0,009)$.

Tabela 3. Médias populacionais das regiões brasileiras, no período de 2008 a 2012, para a cobertura por planos privados de saúde. De acordo com o sexo e faixa etária.

\begin{tabular}{lccccc}
\multicolumn{1}{c}{ Variáveis } & Norte & Nordeste & Sudeste & Sul & Centro-Oeste \\
\hline Sexo & & & & & 23,3 \\
\hline Masculino & 12,7 & 12,4 & 34,7 & 29,1 & 25,9 \\
\hline Feminino & 13,8 & 13,9 & 36,5 & 30,9 & 21,1 \\
\hline Faixa Etária (anos) & & & & & 26,5 \\
\hline 0 a 9 & 10,4 & 10,2 & 32,5 & 26,4 & 25,2 \\
\hline 10 a 19 & 10,9 & 9,9 & 30,3 & 24,6 & 30,0 \\
\hline 20 a 39 & 14,8 & 14,6 & 37,6 & 31,8 & 30,9 \\
\hline 40 a 59 & 17,6 & 17,2 & 36,6 & 33,3 & 31,9 \\
\hline 60 anos ou mais & 15,0 & 15,1 & 38,3 & & \\
\hline
\end{tabular}

*Salário Mínimo (SM): análise com base no salário mínimo de $R \$ 622,00$ correspondente ao período do estudo.

\section{DISCUSSÃO}

$\mathrm{Na}$ presente pesquisa, os usuários das cinco regiões geoeconômicas do Brasil foram analisados visando incluir as diferentes populações, culturas e crenças do mestiço país continental, que possam influenciar no acesso e comunicação com o sistema ${ }^{14}$.

Todas as faixas etárias estão presentes, para que enfermidades mais frequentes em determinados períodos da vida não sejam excluídas, além disso, as idades padronizadas pela OMS para mensuração de alguns índices (5 e 12 anos) também estão em análise ${ }^{15}$.

A população brasileira encontra-se dividida por zona de moradia, urbana ou rural, de forma que a localização e a dificuldade de acesso ao serviço devem ser levadas em consideração no estudo do acesso aos serviços ofertados pelo SUS. Além disso, a renda pode influenciar na saúde, de forma que todos esses 
dados devem ser incluídos, para que sejam contemplados os objetivos de um estudo como o que aqui é apresentado ${ }^{16}$.

Observa-se que o número de ESBs varia entre as regiões brasileiras, porém, ressaltase que a quantidade de municípios, número de unidades da Estratégia Saúde da Família, de usuários e profissionais disponíveis para cada região é diferente.

Não é sensato, que a região Nordeste, com a segunda maior população, e terceiro maior território e PIB, apresente condições socioeconômicas ou até mesmo necessidade de ter disponível a mesma quantidade de ESBs que a região Sul, por exemplo, com menor cobertura territorial e PIB muito maior que o Nordeste ${ }^{17}$. Porém, os dados não especificam a distribuição dessas ESB por estados e municípios e isso é outro fator que pode influenciar no acesso e periodicidade de usuários aos serviços ${ }^{18}$.

A distribuição desses cirurgiões dentistas se mostra de maneira desigual no território brasileiro, majoritariamente, concentrando-se nas capitais e grandes centros urbanos, deixando à deriva os usuários da população rural. Apesar desse aspecto, o fator localização não mostra grandes dificuldades para utilização do serviço público ${ }^{2}$.

Contudo, ainda são escassos os estudos que comprovam o acesso da população rural ao serviço odontológico ${ }^{19}$. Pode-se pressupor que estes usuários utilizam de serviços urbanos para sanar suas necessidades quando necessário, independentemente do tipo, seja ele público ou privado.

A região Nordeste apresenta-se com uma visível iniquidade relacionada à oferta e a utilização dos serviços públicos odontológicos.

Esse fato pode ser provavelmente explicado pelas maiores dificuldades socioeconômicas da região em relação às demais, na qual a população em todas as faixas etárias, com as menores condições financeiras, deve priorizar aspectos como alimentação e moradia, e com isso a saúde bucal passa a ser preferência apenas em momentos de dor ou incômodo. Como resultado, a busca vem a ser por procedimentos reabilitadores e a procura por prevenção permanece ausente ${ }^{20}$.

No Sul, além das maiores médias de ESB, há também um grande número populacional coberto por planos de saúde. Com isso, esta região se destaca por apresentar o menor número de usuários que nunca visitaram o serviço odontológico ofertado pelo SUS.

No entanto, a presença do financiamento privado de saúde pode não explicar a ausência dos usuários do serviço público, pois muitas empresas prestadoras de serviço privado de saúde não ofertam serviços odontológicos aos seus usuários. Com isso, muitos buscam principalmente ações da atenção básica para procedimentos de fácil resolutividade no âmbito público ${ }^{10}$.

Nas demais regiões, pode-se verificar que os resultados se mantêm homogêneos, sendo as mulheres, de baixa renda, das distintas faixas etárias, de moradia urbana, as que nunca se utilizaram dos serviços odontológicos ofertados pelo SUS.

Talvez o acúmulo de funções destinadas ao sexo feminino nos dias atuais, bem como a falta de tempo disponível condizente com os horários de atendimentos dos serviços, além da priorização da saúde dos filhos, principalmente no caso de gestantes, podem ser pontos relevantes que possam justificar tal ausência e periodicidade de busca do cirurgião dentista 21 .

A utilização do serviço público pode ser guiada por diferentes perfis de atendimento, seja ele pautado em métodos preventivos a serem realizados na atenção básica, em métodos curativistas, e até mesmo na utilização como porta de entrada para consultas especializadas, como as ofertadas nos Centros de Especialidades Odontológicas (CEOs) $^{6,22}$.

Esse tipo de serviço oferecido pode influenciar diretamente nos retornos e periodicidade do usuário aos consultórios odontológicos, considerando que a atenção básica deve realizar seu papel de ordenadora do cuidado, conscientizando o paciente na perspectiva de seu acompanhamento e não abandono do tratamento, proporcionando continuidade ao cuidado em saúde e 
possibilitando o funcionamento harmônico do sistema ${ }^{1}$.

Outras medidas preventivas implantadas pela esfera administrativa, como por exemplo, a fluoretação das águas de abastecimento, consolidação de programas educativos nas escolas, visitas domiciliares pelas ESBs e levantamentos epidemiológicos, fazem com que os índices de enfermidades que acometem os usuários tenham regredido e, consequentemente, diminua a busca e a periodicidade das idas aos serviços ${ }^{23}$.

Apesar de todas as mudanças $\mathrm{e}$ conscientização feitas por parte dos profissionais e gestores, a principal busca pelo cirurgião dentista ainda ocorre em quadros de dor, sendo que, embora já se tenha o acesso público ao tratamento endodôntico $\mathrm{e}$ restaurador, ainda são comuns as exodontias, com extensas perdas dentárias sendo verificadas entre os usuários ${ }^{24}$.

Os achados do presente estudo demonstram que a procura por serviços odontológicos é maior entre usuários cobertos por planos de saúde. Tal situação poderia ser atribuída ao fato de estes indivíduos buscarem a atenção em saúde de uma forma geral, compreenderem a importância da saúde bucal, e perceberem necessitar de tratamento odontológico, não sendo ele incluído na maioria das vezes nos planos. Estes usuários procuram o serviço público para atender a suas demandas por atenção em saúde bucal.

0 número de usuários que nunca visitaram o cirurgião dentista se mostra heterogêneo nas distintas regiões do país. Muitas vezes a estruturação dos horários de atendimentos da ESF não condiz com o do paciente, que na maioria dos casos não pode deixar de trabalhar parar ir se consultar, a oferta dos serviços nos turnos noturnos e finais de semana, a facilidade em marcar consulta, se é ou não possível marcá-la em menos de 24 horas ou em tempo hábil para o tratamento, se a marcação pode ser feita através de telefonemas, o tempo de espera para o atendimento, se apresenta equipamentos adequados para diagnósticos e até mesmo medicamentos para o tratamento eficaz, todos estes fatores podem influenciar de forma progressiva ou não para que o acesso da população brasileira à atenção em saúde bucal seja ampliado ${ }^{8,25}$.

Os dados utilizados no presente trabalho são disponibilizados pelo Ministério da Saúde e obtidos de forma rápida a partir de consultas no DATASUS, mas uma das limitações do estudo diz respeito ao fato de que estas informações são dados secundários, que não garantem confiabilidade e precisão dos resultados, uma vez que os casos de erros de notificação ou contabilização não podem ser excluídos.

Apesar de tal limitação, os estudos ecológicos, que fazem uso de dados obtidos a partir de sistemas de informação, não devem ser desconsiderados, pois são relevantes para o planejamento e a organização dos serviços de saúde, possibilitando orientar estratégias e ações de intervenção na perspectiva de ampliar e melhorar a oferta da atenção em saúde bucal e impactar positivamente em indicadores epidemiológicos.

\section{CONCLUSÃO}

Os resultados desse estudo revelam que o acesso e a utilização dos serviços públicos de saúde bucal pela população brasileira, nas diferentes regiões geoeconômicas, bem como a quantidade de equipes de saúde bucal, é desigual, havendo maior procura pela atenção em saúde bucal entre os usuários que possuem plano de saúde privado, que pertencem ao sexo feminino, e são residentes na área urbana.

\section{REFERÊNCIAS}

1. Fonseca LE, Figueiredo MCB, Porto CSBM. Management of Primary Care: a challenge for international cooperation in health. Ciên Saúde Coletiva 2017; 22(7):2287-94.

2. Neves M, Giordani JM, Ferla AA, Hugo FN. Odontologia de cuidados primários no Brasil: da Prevenção ao cuidado integral. J Ambul Care Manage. 2017; 40(Supl 2):35-48.

3. Ministério da Saúde (Br). Portaria no 1.444 de 28 de dezembro de 2000. Estabelece incentivo financeiro para a reorganização da atenção à saúde bucal prestada nos municípios por meio do Programa de Saúde 
da Família. D.O.U., Brasília, DF, 29 dez 2000. Seção 1, p. 85.

4. Ministério da Saúde (Br). Portaria no 267 de 06 de março de 2001. Aprova as normas e diretrizes de inclusão da saúde bucal na Estratégia do Programa Saúde da Família (PSF). Brasilia, DF, n. 119, 7 mar 2001. Secção 1, p. 67.

5. Lino PA, Werneck MAF, Lucas SD, Abreu MHNG. Análise da atenção secundária em saúde bucal no estado de Minas Gerais, Brasil. Ciên Saúde Coletiva. 2014; 19(9):3879-88.

6. Silva HECD, Gottems LBD. The interface between primary and secondary care in dentistry in the Unified Health System (SUS): an integrative systematic review. Ciên Saúde Coletiva. 2017; 22(8):2645-57.

7. Roncalli AG, Côrtes MIS, Peres KG. Perfis epidemiológicos de saúde bucal no Brasil e os modelos de vigilância. Cad Saúde Pública. 2012; 28(Supl1):58-68.

8. Limão NP, Protasio APL, Machado LS, Gomes LB, Valença AMG. Oferta da assistência odontológica especializada na atenção básica do Brasil, Nordeste e Paraíba. REFACS (online). 2017 [citado em 12 jan 2017]; 5(Supl.1):131-40. Disponível em: http://seer.uftm.edu.br/revistaeletronica/in dex.php/refacs/article/view/1987/2029.

9. Ribeiro JM, Moreira MR, Ouverney AM, Silva CMFPD. Políticas de saúde e lacunas federativas no Brasil: uma análise da capacidade regional de prestação de serviços. Ciên Saúde Coletiva. 2017; 22(4):1031-44.

10. Malta DC, Stopa SR, Pereira CA, Szwarcwald CL, Oliveira M, Reis AC. Private health care coverage in the brazilian population, according to the 2013 Brazilian National Health Survey. Ciên Saúde Coletiva. 2017; 22(1):179-90.

11. Instituto Brasileiro de Geografia e Estatística. Pesquisa Nacional de Amostra por Domicílio [Internet]. Rio de Janeiro: IBGE; [201-] [citado em 20 fev 2017]. Disponível em:

http://www.sidra.ibge.gov.br/bda/tabela/lis tabl.asp? $\mathrm{z}=$ pnad \&o=10\&i $=\mathrm{P} \& \mathrm{c}=2494$

12. Lakatos EM, Marconi MA. Fundamentos da metodologia científica. 8 ed. São Paulo: Atlas; 2017.
13. Ministério da Saúde (Br). DATASUS. [Internet]. Brasília, DF: Ministério da Saúde; [201-] [acesso em 12 abr 2017]. Disponível em:

http://www2.datasus.gov.br/DATASUS/inde x.php?area $=02$

14. Peres KG, Peres MA, Boing AF, Bertoldi AD, Bastos JL, Barros, AJD. Redução das desigualdades sociais na utilização de serviços odontológicos no Brasil entre 1998 e 2008. Rev Saúde Pública. 2012; 46(2):250-8.

15. Frencken JE, Sharma P, Stenhouse L, Green D, Laverty D, Dietrich T. Epidemiologia da cárie dentária e periodontite severa - uma revisão abrangente. J Clin Periodontol. 2017; 44 (Supl 18): S94-S105.

16. Perazzo MF, Gomes MC, Neves ÉT, Martins CC, Paiva SM, Granville-Garcia AF. Qualidade de vida relacionada à saúde bucal e senso de coerência quanto ao uso de serviços odontológicos por pré-escolares. Int J Paediatr Dent. 2017; 27(5):334-43.

17. Instituto Brasileiro de Geografia e Estatística. Brasil em síntese [Internet]. Rio de Janeiro: IBGE; [201-] [acesso em 20 jan 2017]. Disponível

em: http://brasilemsintese.ibge.gov.br/territorio 18. Silva CSO, Fonseca ADG, Souza LPS, Siqueira LG, Belasco AGS, Barbosa DA. Integralidade e Atenção Primária à Saúde: avaliação sob a ótica dos usuários. Ciên Saúde Coletiva. 2014; 19(11):4407-15.

19. Cavalcanti RP, Gaspar GS, Goes PSA. Utilização e acesso aos serviços de saúde bucal do SUS: uma comparação entre populações rurais e urbanas. Pesqui Bras Odontopediatria Clín Integr. 2012; 12(1):121-6.

20. Bastos ML, Menzies D, Hone T, Dehghani K, Trajman A. 0 impacto do saúde familiar brasileira em condições selecionadas sensíveis ao cuidado primário: uma revisão sistemática. PLoS ONE. 2017; 12(8): e0182336.

21. Silva VM, Pereira IV, Rocha MJ, Caldeira AP. Morbidade em usuários das equipes de saúde da família no nordeste de Minas Gerais com base na Classificação International de Cuidados Primários. Rev Bras Epidemiol. 2014; 17(4):954-67.

22. Pereira IF, Santiago BM, Oliveira CR, Figueiredo CC, Cunha DA, Sales GLD, et al. 
Evolução da cobertura em saúde bucal na Estratégia Saúde da Família em municípios paraibanos. Rev APS. 2014; 17(1):44-9.

23. Saliba NA, Moimaz SA, Fadel CB, Bino LS. Saúde bucal no Brasil: uma nova política de enfrentamento para a realidade nacional. ROBRAC. 2010; 19(48):66-9.

24. Martins EP, Oliveira OR, Bezerra SRS, Dourado AT. Estudo epidemiológico de urgências odontológicas da FOP/UPE. RFO UPF. 2014; 19(3):316-22.

25. Protasio APL, Machado LS, Gomes LB, Valença AMG. User satisfaction with primary health care by region in Brazil: 1st cycle of external evaluation from PMAQ-AB Ciên Saúde Coletiva. 2017; 22(6):1829-44.

\section{CONTRIBUIÇÕES}

Raphael Cavalcante Costa contribuiu no conceito, delineamento, coleta de dados e redação. Isabella Lima Arrais Ribeiro atuou no delineamento e análise de dados. Larycia Vicente Rodrigues participou na análise de dados. Ana Maria Gondim Valença atuou no delineamento e revisão crítica.

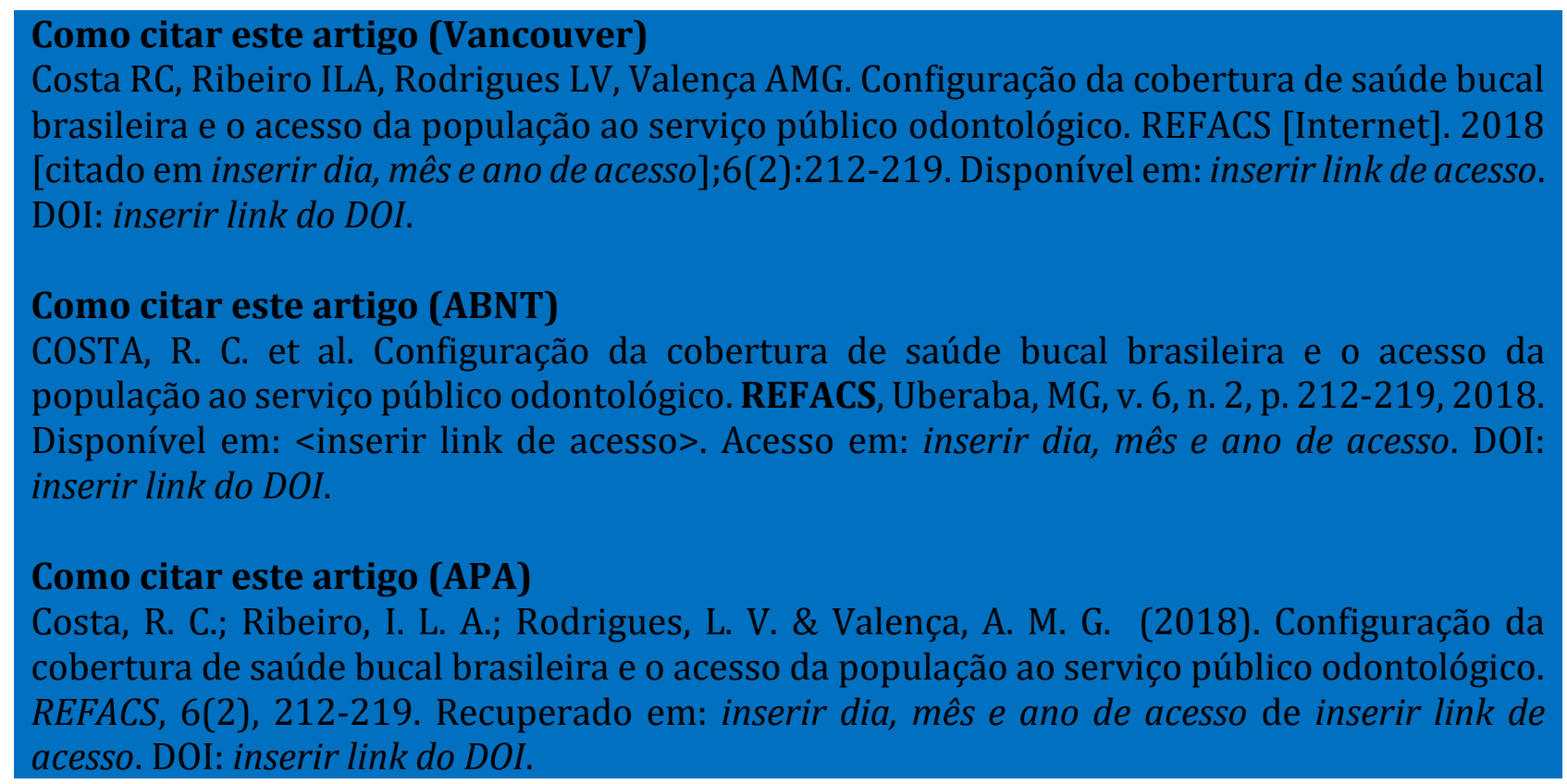

